\title{
Il quarantesimo anniversario della strage alla stazione di Bologna: luoghi di memoria, luoghi non praticabili, luoghi virtuali
}

Il quarantesimo anniversario della strage alla stazione di Bologna si è svolto durante la pandemia, questo ha provocato una rimodulazione della commemorazione ed una discussione pubblica sulla memoria e sul rapporto fra luoghi fisici e luoghi virtuali.

The fortieth anniversary of the massacre at the Bologna station took place during the pandemic. This situation has provoked a remodeling of the commemoration and a public discussion on memory and its relationship between physical and virtual places.

\section{La commemorazione al tempo del Covid 19}

Per la città di Bologna - e non solo - la data del 2 agosto è inserita nel calendario civile e per ciò un momento estremamente importante per la sua comunità è quello della commemorazione ufficiale che si è ripetuta, pressoché immutata nel suo schema, dal 1981. Ogni anno un corteo partiva la mattina del 2 da piazza Maggiore, dopo un incontro nella sala del Consiglio comunale fra i famigliari delle vittime e istituzioni locali, e si dirigeva verso la stazione in una composizione che seguiva sempre lo stesso schema: in testa i famigliari delle vittime, quindi i gonfaloni delle città italiane che portano simbolicamente la partecipazione delle comunità, a seguire i cittadini con la richiesta (quasi sempre rispettata) di non avere simboli o bandiere di partito. Un chiaro esempio, quindi, di pratica sociale che ci mostra un aspetto dell'organizzazione della memoria collettiva di questa strage. 
La mattinata del 2 agosto ha sempre avuto, quindi, il carattere di una commemorazione pubblica con una formula solennizzata e codificata, che di anno in anno ha parlato ad un pubblico uguale e dissimile al tempo stesso. A causa della pandemia il quarantesimo anniversario della strage di Bologna, un anniversario significativo sia per la cifra tonda sia per l'apertura di nuove indagini e nuovi procedimenti penali, ha avuto invece uno svolgimento particolare, diverso da quello ritualizzato. Questa situazione ha suscitato delusioni, polemiche e tensioni, proprio perché si è modificata una pratica consolidata e in molti hanno temuto che a ciò potesse corrispondere una mutilazione della memoria.

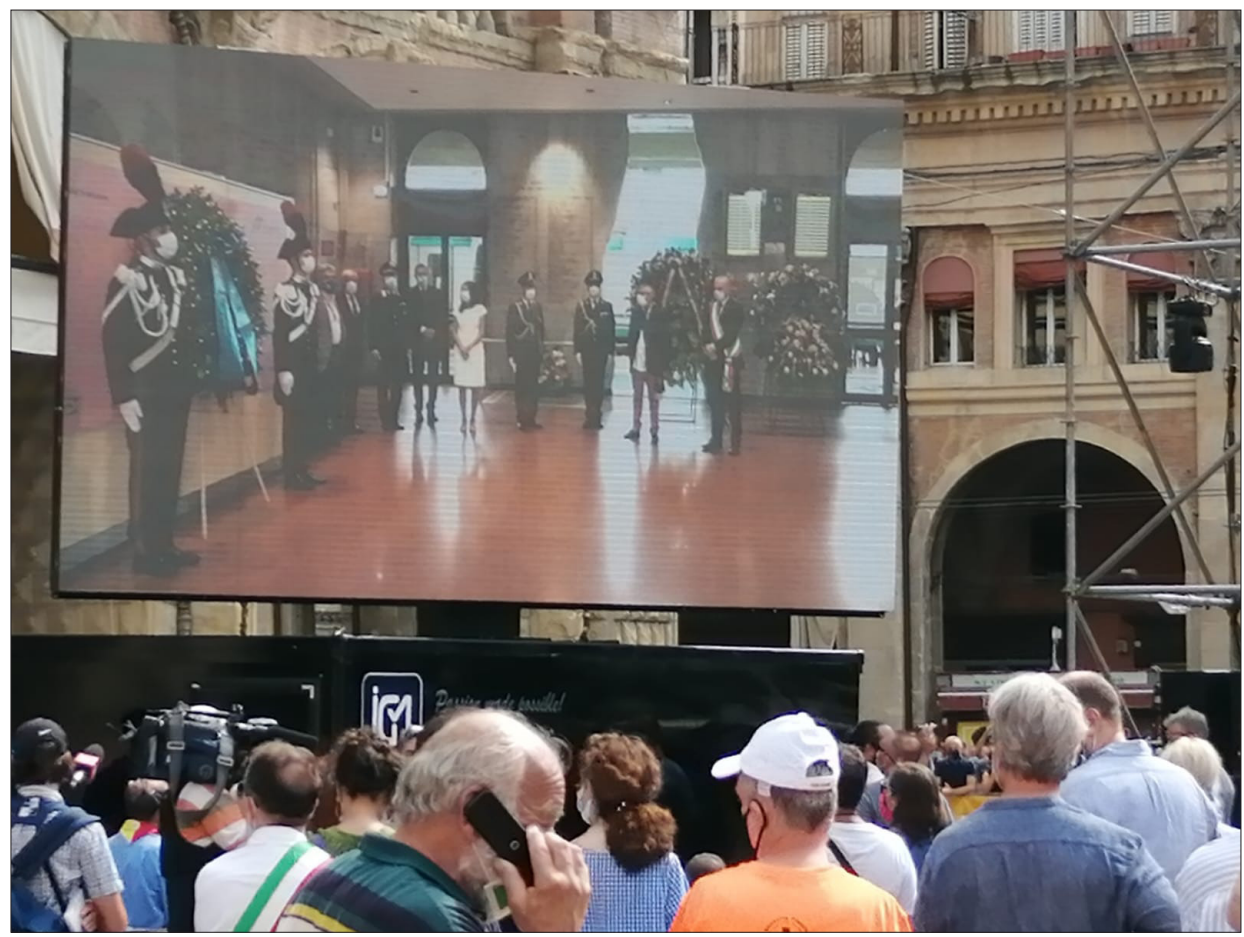

Piazza Maggiore 2 agosto 2020 durante la commemorazione (foto di Agide Melloni).

Le commemorazioni che si sono svolte in questi particolari momenti hanno determinato, in Italia e altrove, discussioni pubbliche sia quando si sono tenute nella prima fase della pandemia, in pieno lockdown (come è accaduto per il 25 aprile), sia in condizioni di restrizioni meno drastiche. A titolo di esempio, si pensi a quello che è recentemente successo in Grecia durante le commemorazioni del 17 novembre, data in cui - con una importanza riservata alle feste nazionali - si ricorda l'irruzione dei carri armati della Giunta di Papadopoulos nel Politecnico di Atene, che provocò morti, feriti, arresti e implicò la sparizioni di studenti e 
studentesse. Quest'anno, causa pandemia, non si sono tenute manifestazioni in presenza e sono state proibiti i cortei che erano, invece, una delle caratteristiche del codice commemorativo. Le polemiche sono state moltissime, fra cui quelle dei deputati di Syriza, Kke e Mera 25 (partiti di opposizione) che hanno condannato «la decisione autoritaria del governo» di vietare la celebrazione del 17 novembre, dichiarando:

La decisione del capo della polizia ellenica, che vieta incontri pubblici di più di tre persone in tutto il Paese e per quattro giorni, sotto la minaccia di multe elevate, ci porta ad altri tempi. Si tratta di una decisione profondamente antidemocratica e incostituzionale, come sottolinea l'Unione dei giudici e dei procuratori, in quanto viola il nucleo del diritto costituzionale di riunione (articolo 11), imponendo una sospensione totale non autorizzata della libertà di riunione e non solo una certa restrizione locale. Tuttavia, un tale divieto generale è imposto solo in condizioni di stato d'assedio (articolo 48 della Costituzione) e in condizioni strettamente limitate. Non può in nessun caso essere giustificato da motivi di tutela della salute pubblica'.

Un corteo organizzato dal partito comunista greco, all'aperto e con i mezzi di protezione individuale per il contrasto della pandemia, è stato caricato dalle forze dell'ordine e vi sono stati feriti ed arresti. Il dibattito si è trasferito sui mezzi di comunicazione di massa e nel Parlamento.

Talmente forte appare in alcuni contesti la carica simbolica della memoria ritualizzata, della memoria culturale praticata nello spazio pubblico, che ogni cambiamento causa - come si diceva - reazioni da parte di chi teme che un mutamento della cerimonia possa ledere il conservarsi e il tramandarsi del ricordo, così che all'annuncio dell'impossibilità di celebrare il 2 agosto nel modo abituale le reazioni sono state molteplici. Pochi giorni prima dell'anniversario, il Comune di Bologna informava i cittadini che non si sarebbe tenuto il corteo che di consueto permetteva alle persone di raggiungere il piazzale della stazione e ascoltare le orazioni commemorative; e che i discorsi del presidente dell'Associazione delle vittime, del Sindaco e della presidente del Senato si sarebbero tenuti in piazza maggiore nelle strutture predisposte per il Cinema in piazza con ingresso contingentato e solo su prenotazione. Il tradizionale minuto di silenzio - anche questo in piazza Maggiore - sarebbe stato preceduto, alle 10.25, dal fischio del treno in collegamento streaming con la stazione; infine, alle 11.15, in piazza Maggiore si sarebbero trasmesse in collegamento streaming dalla lapide nella sala d'aspetto della stazione la deposizione delle corone in memoria delle vittime della strage e 
la scopertura della targa di intitolazione della stazione alle vittime della strage del 2 agosto 1980. In Consiglio comunale, il gruppo Coalizione civica per Bologna invitava «il Sindaco e la Giunta d'intesa con l'Associazione dei familiari delle vittime e la Prefettura, a prevedere lo svolgimento del corteo da piazza Maggiore a piazza Medaglie d'Oro in sicurezza e un accesso contingentato a piazzale di Medaglie d'Oro per il tradizionale fischio della locomotiva delle 10.25 e il minuto di silenzio, che da quarant'anni unisce la città nell'abbraccio ai familiari delle vittime». Molte persone, attraverso il web, si sono mobilitate per chiedere che, pur nel rispetto di tutte le norme anti covid, vi fosse la possibilità di fare il corteo e di conservare così la tradizionale impostazione', temendo che questa nuova struttura della celebrazione, letta come una "diminutio" potesse essere un precedente e paventando che un cambiamento motivato dalla pandemia si potesse trasformare in qualcosa di stabile.

Nei 40 anni che ci separano dalla strage, vi sono stati altri momenti in cui si avanzò l'idea di cambiare la cerimonia, partendo dal 2000, quando iniziarono le contestazioni rivolte ai rappresentanti del governo durante i discorsi tenuti sul piazzale della stazione [Venturoli 2020b]. Anche nel venticinquesimo anniversario vi fu la paventata contestazione a base di fischi nei confronti del ministro Tremonti. I quotidiani del 3 agosto successivo ne parlarono a lungo, per stigmatizzare il «maleducato» e «fazioso» comportamento della piazza bolognese; molti furono gli interventi politici a difesa e in solidarietà di Tremonti, con anche qualche presa di posizione sulla legittimità di potere anche fischiare chi si rivolge da un palco ai cittadini e la proposta di alcuni, fra cui il sindaco Cofferati, di mutare la cerimonia dell'anniversario, magari chiudendo il ricordo all'interno di una sala, in cui tenere un convegno, una riflessione sul terrorismo, allontanandola così dalla piazza, dalla stazione e dallo spazio pubblico. Il presidente dell'Associazione dei famigliari delle vittime sottolineava, però, che non è che c'è qualcosa che non va nella commemorazione del 2 agosto: se dopo 25 anni vengono 10 mila persone, allora vuol dire che è una manifestazione riuscita, che coglie nel segno» ${ }^{3}$.

Non venne elaborata nessuna proposta definita, ma quattro anni dopo dalle pagine di "la Repubblica", edizione Bologna, si proponeva di «uscire dal rito di discorsi e corteo», visto che - secondo la testata - si sentiva la necessità di cambiare e di superare la mera commemorazione ${ }^{4}$. L'ex sindaco Giorgio Guazzaloca, come ri-

Strage diBologna, pernon dimenticare, "GlobalistSyndication", 21 luglio 2020 [https://www.globalist.it/news/2020/07/21/ strage-di-bologna-per-non-dimenticare-2062158.html]

"la Repubblica", 8 aprile 2005.

"la Repubblica", 28 luglio 2005. 
portava lo stesso giornale, il 2 agosto 2009 chiese al presidente dell'Associazione famigliari delle vittime Paolo Bolognesi di farsi da parte: «Consiglio a Bolognesi un'uscita strategica dalla manifestazione in onore delle vittime del 2 agosto. Si presenti domattina dal sindaco e rimetta a lui e ai presidenti di Provincia e Regione la decisione di come, a cominciare dal prossimo anno, rinnovare una "liturgia" che si ripete da 29 anni» e il deputato del Popolo delle libertà Giuliano Cazzola rincarava la dose, affermando che nel trentesimo anniversario «le vittime del 2 agosto le commemoreremo per conto nostro». «Abbiamo bisogno di rimettere in gioco rituali che ci danno un grande senso di identità, come è giusto che sia, ma che non soddisfano più lo scopo principale per cui erano pensati» affermava il Sindaco Delbono dal palco del piazzale della stazione $e^{5}$, sulla stessa lunghezza d'onda, la Commissaria prefettizia Annamaria Cancellieri, che sostituì l'anno successivo il dimissionario Delbono.

Una manifestazione che invece non mutò la sua struttura, come si è detto, ma che fu di anno in anno preceduta, affiancata e seguita da iniziative che avevano l'intenzione di utilizzare linguaggi in grado di coinvolgere pubblici diversi non solo nella trasmissione di memoria, ma anche nell'approfondimento della conoscenza

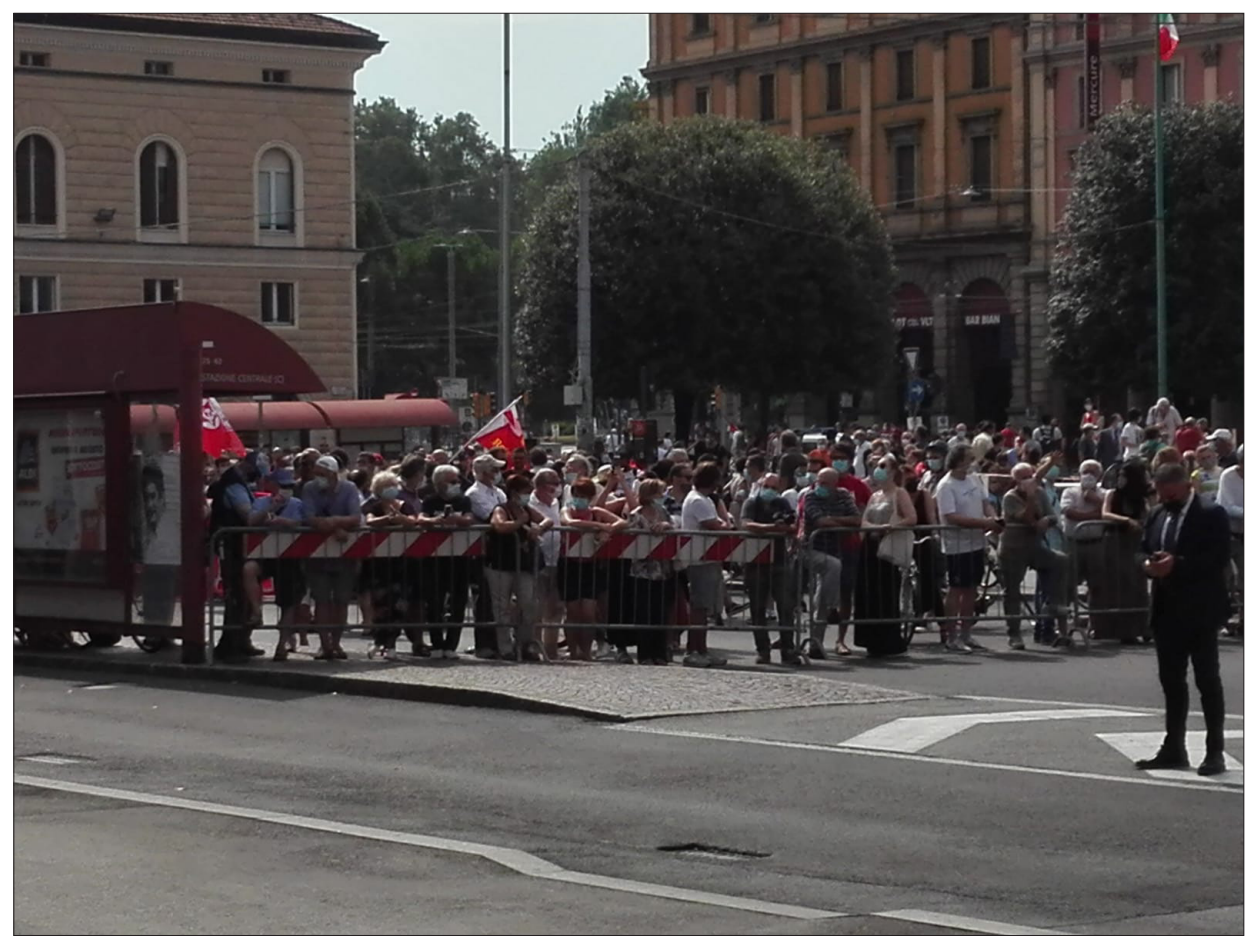

2 agosto 2020, piazzale della stazione (foto di Gianni Zini). 
storica in progetti che hanno visto nella public history un supporto ed uno sviluppo prezioso ${ }^{6}$, alla luce delle necessità della contemporaneità in cui memoria $\mathrm{e}$ storia devono essere in grado di dialogare in modo proficuo senza annullarsi l'una nell'altra [Venturoli 2020a].

Dei cambiamenti decisi per il quarantesimo anniversario, quello che più ha fatto discutere è stata l'impossibilità di raggiungere in una forma collettiva la stazione, tanto che un corteo è stato fatto comunque e molte persone si sono dirette, anche autonomamente, nel piazzale della stazione, sentendo la necessità di essere nel luogo di memoria, punto di condensazione della memoria, dove sono i segni che rendono "visibile" il passato e dove ci si sente in empatia con le vittime: il non poter essere in quel luogo ha portato con sé lo spaesamento, di cui si è detto. La presenza di lapidi e di scritte, di immagini e di segni si rifà a una concezione antica della memoria: la memoria divinatoria pensata fin dalle radici della filosofia e delle culture, che si crede durare solo in presenza di quegli oggetti. Forse anche per questo motivo, la loro rimozione, il loro danneggiamento, la loro mutazione, l'impossibilità di presidiare - almeno durante l'anniversario - lo spazio che li contiene, provoca in chi quella memoria vuole che continui ad esistere sgomento e il timore che, spariti quegli oggetti, abbandonati quei luoghi, con essi scompaia anche la memoria della strage e delle vittime. D'altro canto, è stato più volte rilevato come il potenziale divulgativo, didattico e pedagogico del luogo di memoria, soprattutto del luogo evento, risulti "formidabile" sia per il racconto pubblico sia per una didattica partecipata, prestandosi all'attivazione di numerosi nodi di senso e di competenze - per questo motivo i luoghi di memoria vengono sempre più inseriti all'interno dei percorsi scolastici. Le pratiche sociali di memoria (fra cui monumenti, lapidi e cerimonie commemorative) non rispondono quindi alla sola esigenza di "oggettivare" e istituzionalizzare una data immagine del passato, ma anche a quella di renderla credibile e legittima.

\section{Luoghi virtuali e luoghi fisici}

Fin dal 9 maggio 2020, non potendone prevedere l'evoluzione ma con la certezza che sarebbe stato un anniversario diverso dagli altri, si sono cercati anche luoghi virtuali in cui tenere viva l'attenzione sul quarantesimo anniversario della strage di Bologna: come, ad esempio, la pagina Facebook Dammi la mano Bologna (anche

Fra le iniziative degli ultimi anni, si veda, a titolo d'esempio: Cantiere 2 agosto - 2017 [https://www.assemblea.emr.it/ cantiere-due-agosto/cantiere-due-agosto/il-docufilm]; Piantiamo la memoria - Commemorazione [http://www.piantiamolamemoria.org/category/commemorazione/]. 
in questo 2 agosto), realizzata in collaborazione con l'Associazione dei familiari delle vittime per creare una «una piazza virtuale, che si affianca a quelle reali, per ricordare e conoscere la strage alla stazione di Bologna», dove con la riproposizione di materiali legati a progetti precedenti, con la creazione di nuovi prodotti e con le organizzazione di dirette si è cercato di accompagnare verso il 2 agosto; oppure spazi virtuali occupati nella giornata dello stesso 2 agosto dalla maratona social Artisti per il 2 agosto, promossa dall'Assemblea legislativa della regione Emilia-Romagna e realizzata dal suo Ufficio stampa. Iniziative che hanno avuto un importante seguito con numerosissime visualizzazioni, da parte di persone che quasi certamente non si sarebbero recate a Bologna per la commemorazione, ma che hanno ritenuto di usufruire dello spazio virtuale?

Lo spazio di internet, i social, il web 2.0 da molto tempo sono occupati da una mole rilevante di presenze di "storia e memoria", con diverse modalità di espressione e differenti motivazioni, in questi spazi chiunque può dedicarsi al passato in rete: dai blog, alle pagine Facebook, a Twitter, ai siti. Nei casi succitati promotori sono stati l'Associazione dei familiari delle vittime e l'Assemblea legislativa regionale, che hanno tentato di trasporre in spazi virtuali l'attenzione, quasi a sostenere una memoria - ora privata di spazi reali - attivando e sperimentando nuove modalità di ricordo collettivo, che possono, esse stesse, entrare a fare parte di quel codice stabile di cui le commemorazioni si nutrono e che al momento si rivelano necessarie, in quanto le uniche in grado di garantire una eco sufficientemente ampia quando i luoghi fisici risultino impraticabili.

Per confermare la solennità del quarantesimo anniversario della strage della stazione e di quella di Ustica, invitato dal Sindaco, il 30 luglio 2020 il presidente della Repubblica si è recato in visita a Bologna e ha visitato tre luoghi: la cattedrale di San Pietro, per una messa celebrata dal Cardinal Zuppi; il Museo della memoria di Ustica e la stazione. Oltre alle autorità locali, solo una delegazione delle persone direttamente colpite dalle stragi era presente nei luoghi visitati da Sergio Mattarella: in una sala d'aspetto chiusa al pubblico (dal lockdown ad ora), primo presidente dopo Sandro Pertini a intervenire alla commemorazione della strage, Mattarella ha sottolineato come la sua presenza simboleggiasse «partecipazione al dolore che rimane, [la] solidarietà della repubblica per questo dolore. Il dovere del ricordo della memoria perché non si smarrisca mai la consapevolezza di quanto avvenuto e che va impedito per il futuro». 
Per il quarantesimo della strage di Bologna altri spazi fisici sono stati occupati da iniziative pubbliche: ad esempio, da tre murales inaugurati in contemporanea a Bologna, Parma e Reggio Emilia il 31 luglio, prima parte del progetto curato dell'associazione Serendippo (in collaborazione con Associazione familiari delle vittime, Assemblea legislativa, Fondazione Rusconi e Tper), che prevede la realizzazione di murales in tutti i capoluoghi della regiones.

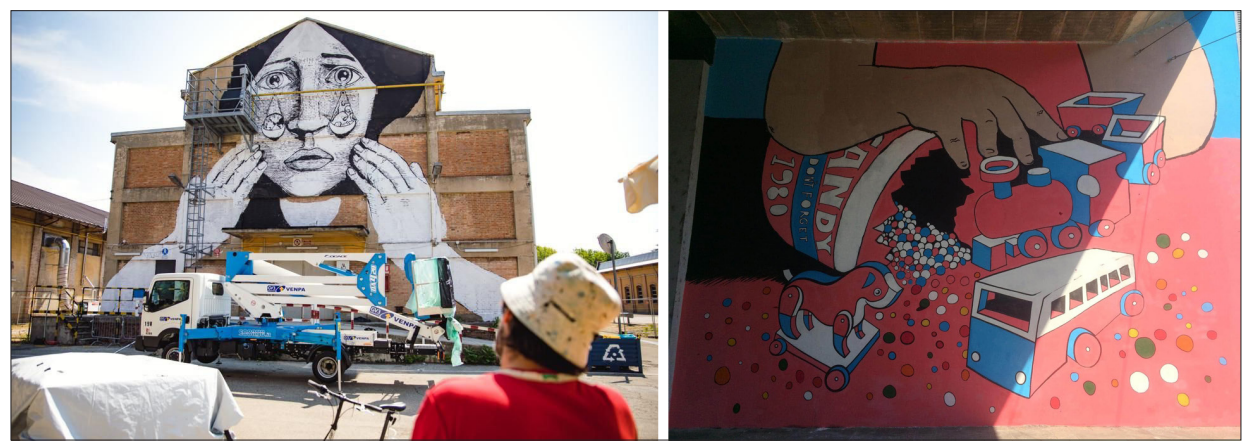

Murales realizzati a Bologna da Collettivo FX e a Parma da Alessandro Canu (foto Archivio Associazione fra i famigliari delle vittime della strage alla stazione di Bologna).
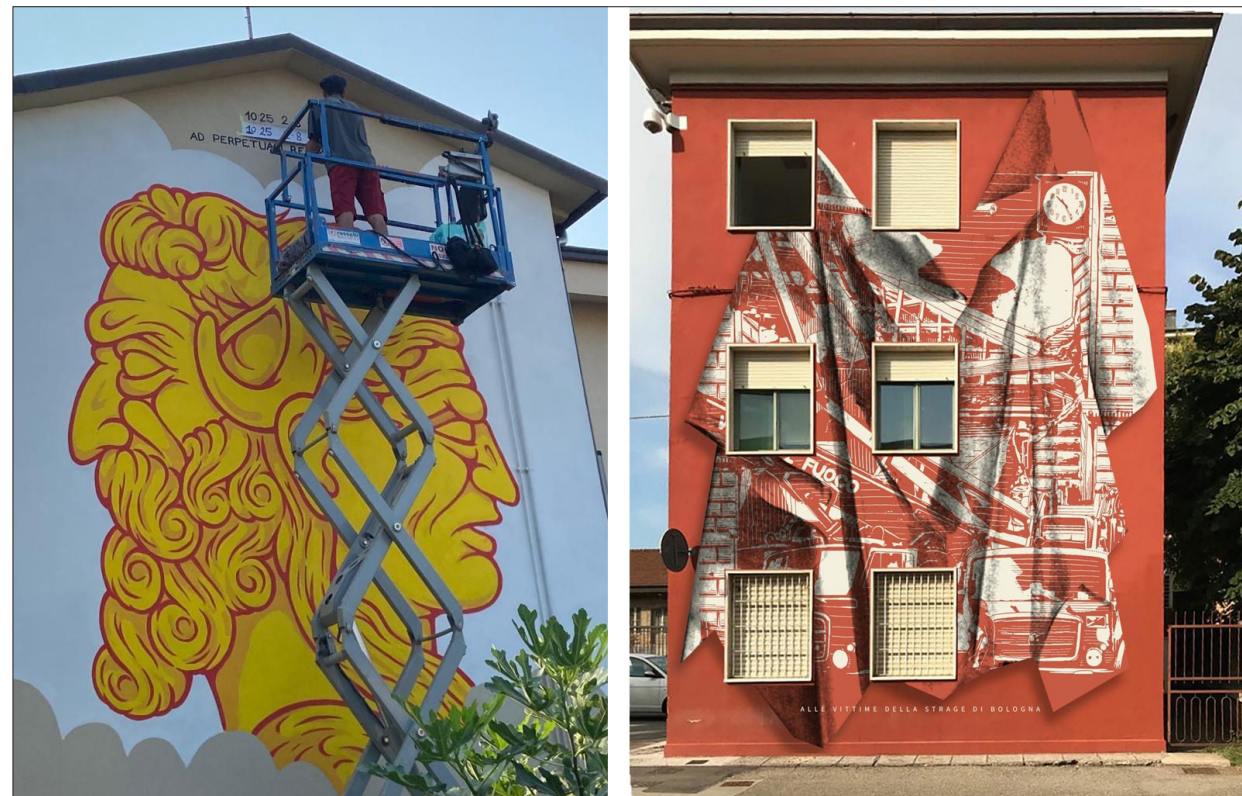

Murales realizzati a Reggio Emilia da PsikoPlanet e a Modena da Zamoc (foto Archivio Associazione fra i famigliari delle vittime della strage alla stazione di Bologna).

8 Lostefound 1980-2020. Memorie private e collettive 40 anni dopo [https://www.assemblea.emr.it/cantiere-due-agosto/lostfound-1980-2020]. 


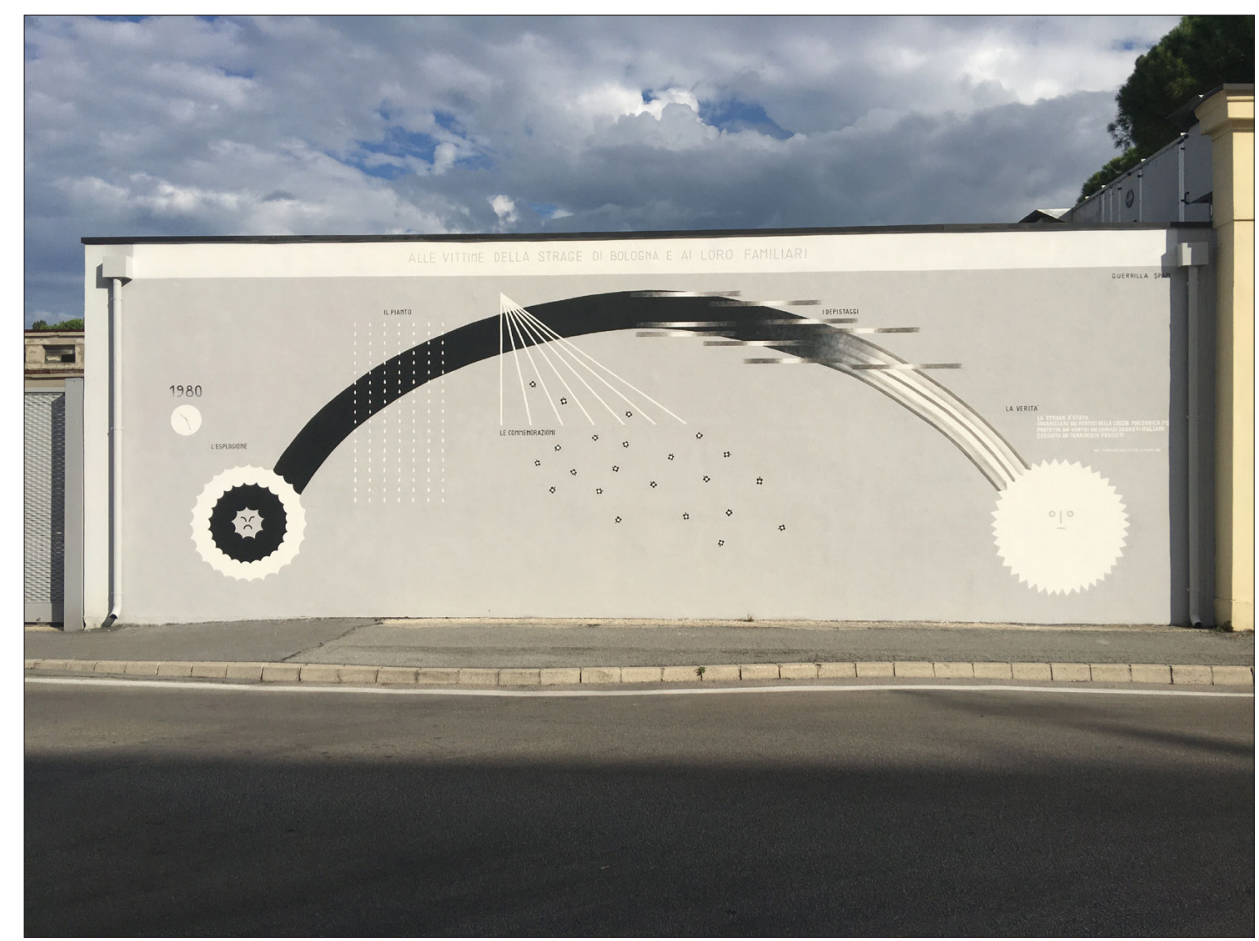

Murales realizzato a Rimini da Guerrila Spam (foto Archivio Associazione fra i famigliari delle vittime della strage alla stazione di Bologna).

Altro spazio investito dalle celebrazioni è stato lo stadio di Bologna, dove in coincidenza con l'ultima partita di campionato (prolungato causa covid), è stato tenuto - seppur a stadio vuoto - in diretta televisiva un minuto di silenzio, anche in questo caso in un'alternanza fra spazi fisici e spazi virtuali.

Se le strade italiane non sono state percorse dalle staffette podistiche che solitamente convergono a Bologna la mattina del 2 agosto, molto seguita è stata l'iniziativa che ha visto il centro della città "occupato" da "Stazioni della memoria del 2 agosto 1980", organizzata da Cantiere Bologna insieme a 6000 sardine e Cucine popolari, e resa possibile dalla collaborazione di centinaia di cittadini che hanno dato un contribuito utilizzando la piattaforma "Ginger" (ed altri sostenitori, fra cui Spi-Cgil Emilia Romagna e Bologna, Hera, Tper, Legacoop, Coop Alleanza.3, Cotabo, Fondazione Unipolis). Il flash mob Station to station, organizzato da "retata di sardine" e da singoli cittadini, ha infine portato nella mattina del 2 agosto alla realizzazione di un simbolico presidio nelle stazioni di città e paesi di differenti regioni italiane.

Ulteriori progetti, quindi, frutto dell'iniziativa di associazioni e cittadini mossi dall'esigenza di presenziare comunque fisicamente all'anniversario, in risposta alla particolarità del momento in cui esso si è svolto: particolarità riconducibile 


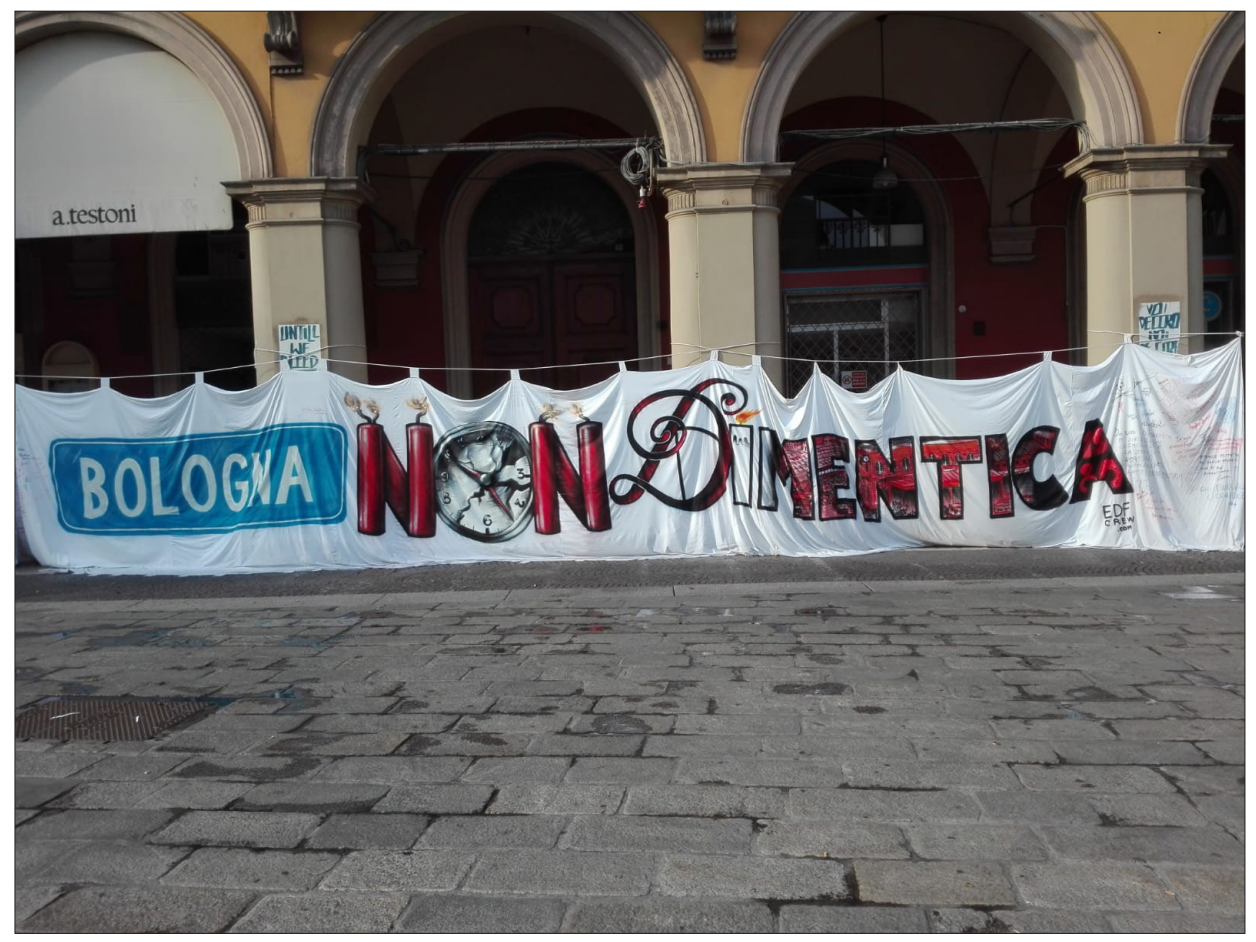

Una delle stazioni di memoria realizzate a Bologna

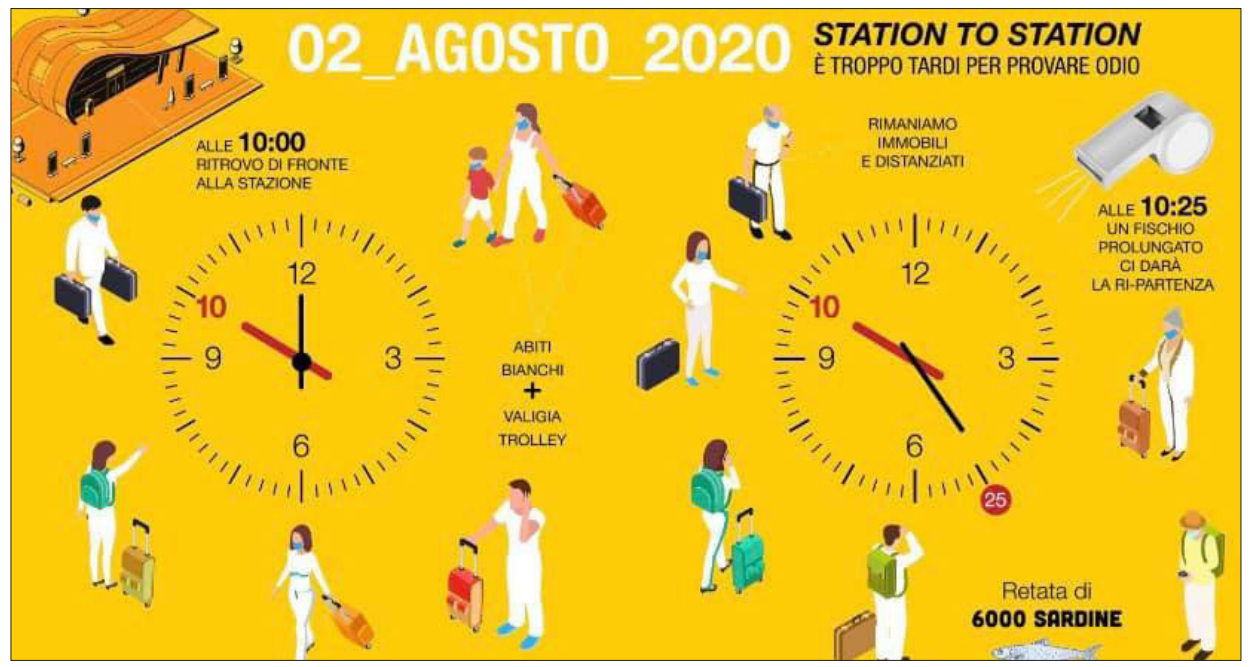

Convocazione flash mob Station to station 


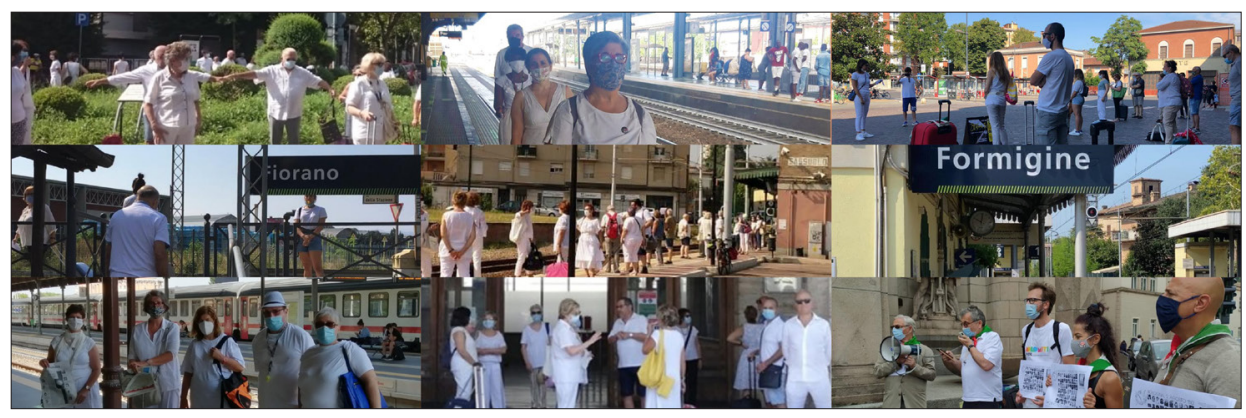

Alcuni dei flash mob Station to station

sia alla pandemia, sia alla ripresa dei procedimenti penali. Come è noto infatti già nel 2011, e poi nel 2015, 1'Associazione dei familiari delle vittime ha consegnato alla Procura di Bologna alcune memorie in cui erano state raccolte informazioni e elementi di prova che, nell'intenzione dei redattori, avrebbero permesso di approfondire le conoscenze processuali sulla strage fino ad arrivare a individuarne mandanti e finanziatori: un risultato ottenuto grazie alla digitalizzazione di un gran numero di documenti processuali e all'utilizzo di strumenti tecnologici per la ricerca all'interno dell'immensa mole di materiali raccolti (Moroni, 2017; Moroni e Tobagi 2018). Il 26 ottobre 2017 il procuratore generale ha avviato una nuova inchiesta e l'11 febbraio 2020 sono stati notificati quattro avvisi di conclusione indagine. Parallelamente il 21 marzo 2018 si è aperto un nuovo filone processuale sui fatti del 2 agosto, che il 9 gennaio 2020 ha portato alla definizione di una condanna in primo grado per complicità nella strage. Queste nuove indagini hanno riportato la strage sulle pagine dei giornali - per quanto resti l'impressione che la stampa e le televisioni nazionali non le abbiano riservato la medesima attenzione di quelle locali - e naturalmente sul web, dove si affollano notizie, siti e blog, pagine Facebook, caratterizzate da una forte presenza di tesi revisioniste e "innocentiste" nei riguardi dei condannati in via definitiva (con la riproposizione di piste alternative già più e più volte prese in esame dalla magistratura e una forte eco fra i banchi della destra parlamentare, sebbene le nuove indagini e l'ultima sentenza emanata indirettamente confermino i giudizi definiti dalle sentenze già passate in giudicato). Presenze innocentiste che sono passate dai già ampiamente presidiati - ora e nel passato - spazi virtuali a quelli reali: il 2 agosto 2020, infatti, in 40 città italiane sono state convocate manifestazioni da parte di chi sostiene l'innocenza dei condannati in via definitiva, con l'intento di negare il coinvolgi- 
mento del terrorismo neofascista nella strage ${ }^{10}$. Così come è capitato in passato - in date cardine quali gli anniversari o durante i dibattiti processuali - anche nel 2020 si è quindi riproposta una discussione pubblica sulle risultanze processuali dai toni a tratti molto aspri.

Luoghi di memoria, luoghi non praticabili, luoghi virtuali hanno quindi dialogato, non senza qualche difficoltà, in questo quarantesimo anniversario, che ha posto - fra gli altri - anche interrogativi sul rapporto fra storia, memoria e public history in un contesto non facilmente gestibile di impraticabilità o limitazioni degli spazi sociali.

\section{Bibliografia}

Moroni I. 2017. Terrorismi e mafie: una storia ancora da scrivere, "Il mondo degli archivi”, 8 maggio 2017 [http://www.ilmondodegliarchivi.org/rubriche/il-potere-degliarchivi/498-terrorismi-e-mafie-una-storia-ancora-da-scrivere-9maggio]

Moroni I., Tobagi B. 2018. La «Direttiva Renzi» e le carte sulle stragi, «Le Carte e la Storia», 2, dicembre

Scardova R. 2016. La strage di Bologna presenta il conto, in Bolognesi P. (ed.) 2016, Alto tradimento. La guerra segreta agli italiani da piazza Fontana alla strage della stazione di Bologna, Roma: Castelvecchi, 9-65

Venturoli C. 2020a. Raccontare il 2 agosto 1980: storia, memoria, public history in Bertella Farnetti P. e Dau Novelli C.(eds.) La storia liberata. Nuovi sentieri di ricerca, Milano: Mimesi Edizioni

Venturoli C. 2020b. Storia di una Bomba Bologna 2 agosto 1980: la strage, i processi, la memoria, Roma: Castelvecchi 